\title{
Exploring the effects of CDK7 inhibition by YKL-5-124 on the molecular mechanisms and the ways it triggers cytokines production in Small Cell Lung Cancer in vitro
}

\author{
Weining Chen ${ }^{1}$ \\ ${ }^{1}$ Biochemistry and Molecular Biology; Department of Biological Science; University of California, Davis; Davis, California, 95616, \\ United States
}

\begin{abstract}
The previous study has researched that combining YKL-5-124, CDK7 specific target drug with anti-PD-1 treatment had a survival advantage in various Small Cell Lung Cancer models. However, the molecular mechanism that YKL-5-124 triggers inflammatory response remains unknown. This study investigates the effects of CDK7 inhibition by YKL-5-124 on two DNA damage pathways (ATM and ATR) of SCLC in vitro. The result of this study will reveal the effects of CDK7 inhibition by YKL-5-124 on the molecular mechanisms of SCLC and potentially promote the development of new anticancer treatment strategies based on immunity manipulation.
\end{abstract}

\section{Introduction}

The small cell lung cancer (SCLC) is one of the most malignant types of lung cancer, characterized by rapid progression and easy to relapse. SCLC is sensitive to chemotherapy and radiotherapy, whereas, for the high recurrence rates of SCLC, there are still many treatment challenges [1]. In recent years, studies have shown that cell cycle regulation kinases also participate in malignant cell transformation [2].

Cyclin-dependent kinases (CDK), protein kinases [3], regulate the cell cycle's initiation and progression, controlling cell proliferation. In particular, CDK7 plays a dual role in both cell cycle control and transcription regulation [4-6].CDK7, cyclin $\mathrm{H}$, and Mat1 form CDKactivated Kinase (CAK). CAK up-regulates cell cycle progression through threonine phosphorylation on cycling-related kinases, such as CDK1/2 [4]. Meanwhile, CAK is one of the subunits of the transcription factor TFIIH that phosphorylate the Ser-5/7 of repetitive Cterminal domain of RNA pol II, allowing promoter clearing [3][7]. However, the role of CDK7 kinase activity in transcription is controversial [8-11]. Zhang et al. and Olson et al. demonstrated YKL-5-124, a drug targeting CDK7, does not significantly inhibit basal transcription $[10,11]$.

YKL-5-124, a drug explicitly targeting CDK7, forms a covalent bond with $\mathrm{C} 312$ on CDK7 that cannot phosphorylate CDK1/2 and down-regulates the cell cycle [10-12]. Interestingly, applying YKL-5-124 to Jurkat cells did not induce apoptosis. YKL-5-124 inhibited CDK1/2 phosphorylation in Jurkat cells at a different level. [11]. Furthermore, Zhang et al. demonstrated YKL-5-124 triggers micronucleus formation and immune response signaling in SCLC. Combining YKL5-124 with anti-PD-1 treatment provides a theoretical basis for a new combination therapy comprising CDK7 inhibitors and immunotherapy [10]. However, the exact molecular mechanisms induced by CDK7 inhibition by YKL-5-124 in SCLC lead to the production of inflammatory factors that remain unknown. It is noteworthy that not just phagocytes and lymphocytes can secrete pro-inflammatory cytokines. SCLC cells also have the ability to secrete inflammatory cytokines in which IL- 8 and TGF- $\beta$ are the major cytokines being secreted. Moreover, neither IL-4 nor TNF- $\alpha$ secretion was detected in lung cell lines [13]. Cell senescence and damage may be accompanied by increasing the expression of the senescence-associated secretory phenotype (SASP), including IL-6, IL-8, TGF- $\beta$, CXCL10, etc. (Figure 1) [14]. Accordingly, YKL-5-124 has the potential to trigger SCLC cells' senescence stress response by attenuating inhibition on CDK2 and leading to the genome instability that secret the inflammatory cytokines via SASP through unknown molecular mechanisms [15]. These unknown molecular mechanisms might be DNA damage repair (DDR) pathways [14][16]. Therefore, unmasking the effects of CDK7 inhibition by YKL-5-124 on SCLC's molecular mechanisms will potentially promote the development of new anticancer treatment strategies based on immunity manipulation.

This study will discuss the necessity of ATM and ATR signaling pathways to repair DNA damage caused by CDK7 inhibition by YKL-5-124 in purified SCLC cells and trigger inflammatory cytokines secretion via inducing the SASP (Senescence-associated secretory phenotype) factor will be discussed. Meanwhile, the generation of micronucleus should be considered as only

cwnchen@ucdavis.edu 
a representation of DNA damage in the inhibition process.

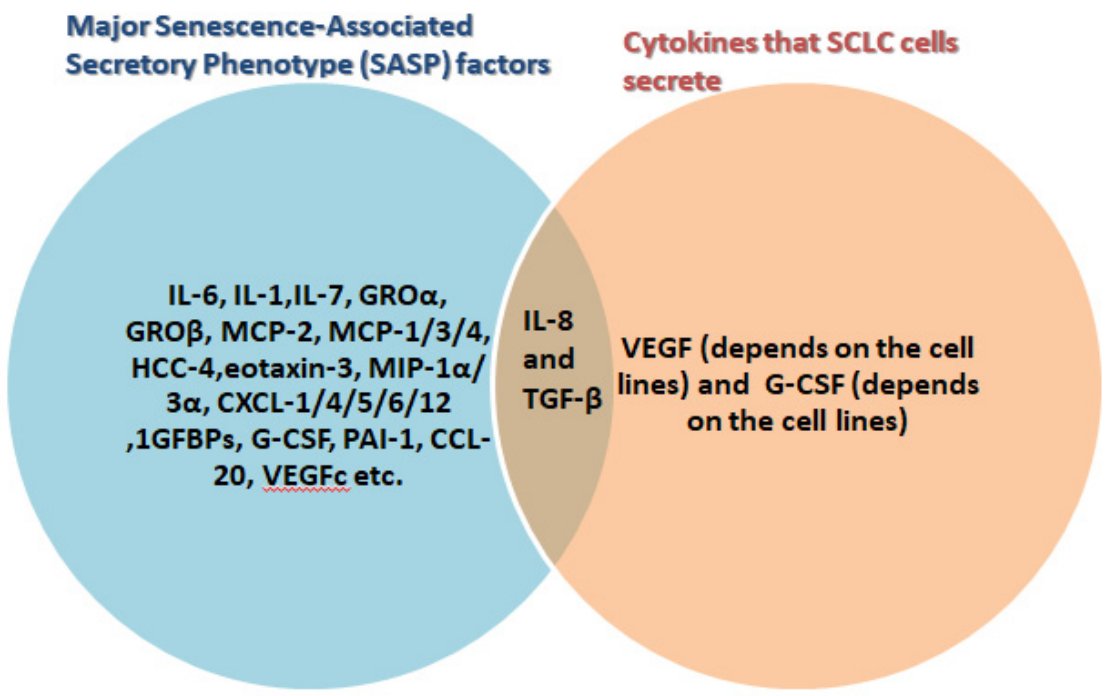

Fig.1 Summary of major SASP factors, and the cytokines secreted by SCLC cells, and the cytokines they have in common [13, 14] [17].

\section{Method}

To estimate the error and variation of the experiment, experiments will be repeated at least three times in this proposal.

\section{Cell Lines} ensure the observation value's reproducibility, and avoid the abnormal results caused by accidents, the

Table 1 Different cell lines will be used in study

\begin{tabular}{|c|c|c|}
\hline Cell Line & Organism Type & Sources \\
\hline HAP1 & Human & Horizon Discovery \\
\hline GLC16 & Human & In previous study [12] \\
\hline DMS79 & Human & In previous study [12] \\
\hline NCI-H82 & Human & In previous study [12] \\
\hline HEK-293T & Human & ATCC \\
\hline NCI-H69 & Human & In previous study [12] \\
\hline RPP631 & Mouse & In previous study [10] [10] \\
\hline RPP-MYC & Mouse & In previous study [10] \\
\hline RP & Mouse & \\
\hline
\end{tabular}

\section{Chemicals}

YKL-5-124

\section{Flow cytometry (FACS)}

FACS instrument is used for SCLC cells (Table 1) sorting. SCLC cells are incubated with unique biomarkers in both lymphocytes and phagocytes (Table 2 ). The antibodies that recognized lymphocytes are conjugated to blue fluorophore and antibodies that recognized phagocytes are conjugated to a red fluorophore to gate cells according to their light scattering characteristics by FlowJo. The magnetically labeled lymphocytes and phagocyte cells are retained in deflection plates. The charged cells are then separated into collection tubes, while the unlabeled SCLC cells continued through and collected in another container [18].

Table 2: Lymphocytes and Phagocytes Biomarker [19-31].

\begin{tabular}{|c|c|c|c|}
\hline \multicolumn{2}{|c|}{ Lymphocytes } & \multicolumn{2}{c|}{ Phagocytes } \\
\hline Type & Marker & Type & Marker \\
\hline B cells & CD19 & Myeloid Progenitor cell & CD33 \\
\hline $\begin{array}{c}\text { Regulatory T cell,T helper cells, } \\
\text { cytotoxic T cells }\end{array}$ & CD3+, CD4+ and & Erythroblast and Eosinophil & $\begin{array}{c}\text { CD71 and } \\
\text { CD44 }\end{array}$ \\
\cline { 2 - 4 } & CD8+ & Megakaryocyte & CD61 \\
\hline NK cell & CD56 & Basophil, monocytes,mast cell & CD123 \\
\hline Plasmacytoid Dendritic Cell & CD303 & Neutrophil & CD15 \\
\hline Plasma Cell & CD138 & Myeloid dendritic cells & CD1c \\
\hline
\end{tabular}

\section{ATR and ATM knockdown cell lines}

shRNAs are used to knockdown ATR and ATM in SCLC human and mouse cell lines by targeting different ATR and ATM gene regions [32]. shRNA oligonucleotides are generated by using the pLenti4/V5DEST vectors (Addgene plasmid \# 42651) [33]. ATR: In human cell lines, the exon 8 (sense sequence CCGCTAATCTTCTAACATT) is targeted. In mouse 
cell lines, the ORF \#14(sense sequence: GCTAACAAGAGCTTACCTTGC) is targeted. ATM: In human cell lines, NTs started at \#1762 (sense sequence: GCAGTATGCTGTTTGACTTTG) is targeted. In mouse cell lines, NTs started from \# 1873 (Mature sense sequence: GCTCTTATTCTACCAGTTAGA) is targeted.

\section{Western blotting}

Equal amounts of SCLC protein samples are loaded for SDS-PAGE and analyzed by gel blot. Primary and Secondary antibodies are used for specific proteins detection. Tubulin is probed to confirm the targeted proteins are extracted from the whole cell lysate [32].

\section{Cell Viability}

Cells are treated with YKL-5-124 at 100nM and measured by CCK-8 assay.

Cell Cycle Analysis

Cells are treated with YKL-5-124 at 100nM and analyzed by flow cytometry [10].

\section{BrdU Analysis}

Cells are treated with YKL-5-124 at 100nM. SCLC cells are analyzed by BrdU kit and gated for FITC and 7AAD [10].

\section{Immunofluorescence Staining and Imaging}

Cells are fixed, and cell nuclei are stained with DAPI. Micronucleus is identified by a microscope [10].

\section{Multiplex Luminex ${ }^{\mathrm{TM}}$ Protein Assays (Thermo}

\section{fisher)}

The Human and mouse Immune Monitoring 65 or 48Plex ProcataPlex Panel (Cat\#EPX650-10065-901 and Cat\# EPX480-20834-901 ) are used to analyze 65 protein targets in each SCLC cell line using Luminex xMAP technology. Analyze 65 or 48 cytokine, chemokine, and growth factor targets simultaneously for efficient immune response profiling, biomarker discovery, and validation.

\section{RNA Extraction and RT-qPCR}

The extracted SCLC RNAs are transcribed into cDNA reversely. cDNA samples are used for RT-qPCR. Gene-specific primers of specific cytokines with sequences are used for PCR amplification and [10].

\section{ELISA}

The levels of immunoreactivity of various cytokines are measured by ELISA [13].

\section{Statistical analysis}

One-way (only ATM or ATR knocked-down) and two-way ANOVA are used for significance tests $(95 \%$ confidence interval), and the comparison can be performed in GraphPad Prism 8. Two-way ANOVA is performed to test the hypothesis that both ATR and ATM are knocked-down [32, 33].

Illustration Tool

The predicted figures are created with Lucidchart and BioRender.

\section{Possible Results}

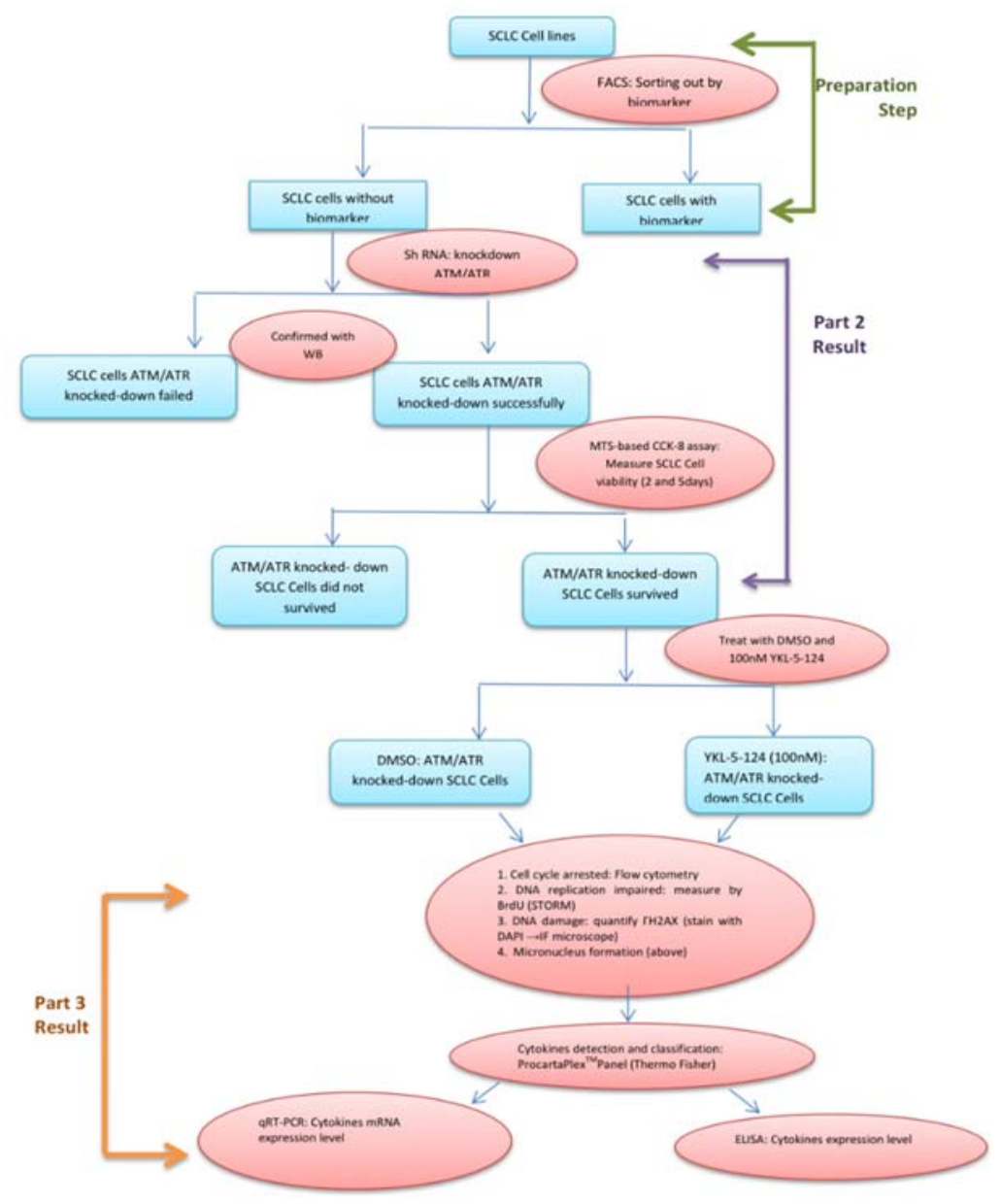

Fig.2 Experimential Design Flow Chart ("blue box" represent SCLC cell samples; "Pink ellipse" represents the method used) 


\subsection{Preliminary experiment: Same results as previous studies (Fig.3)}

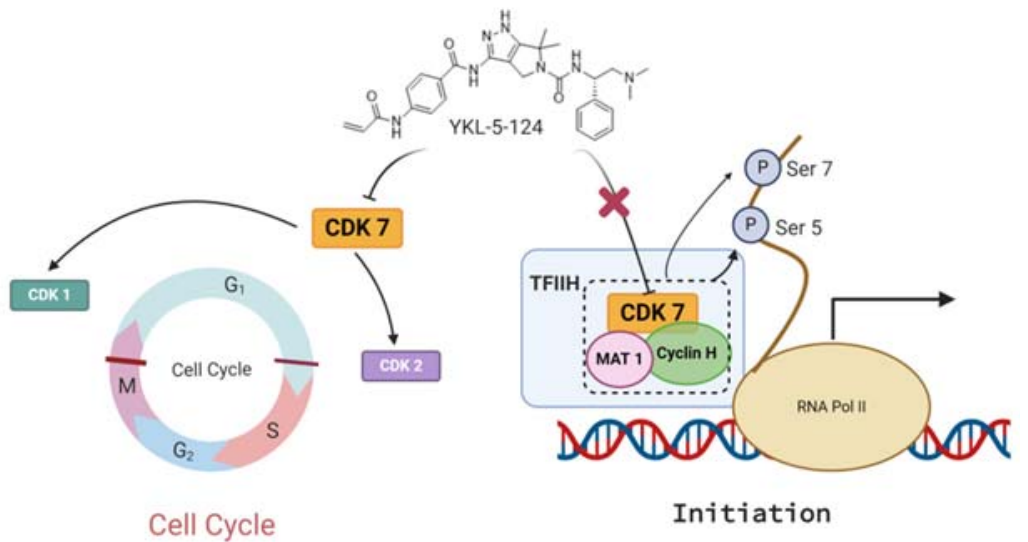

Fig.3 Graphical illustration of previous studies results $[10,11]$

Consistent with recent studies [10,11], in all the cell lines samples, YKL-5-124 induced cell accumulation in G1phase and loss of S-phase cells. Concurrently, Edu stained S-phase cells decreased significantly, suggesting DNA replication inhibition. In fact, after the occurrence of DSBs, phosphoinositide-3 kinase (PI3K) family members rapidly gathered and formed the $\gamma \mathrm{H} 2 \mathrm{AX}$ via phosphorylation at the site of DNA fragmentation, which expanding the signal cascade in DNA damage stress response. This DNA damage stress response can occur in a few minutes, so it is considered one of the most

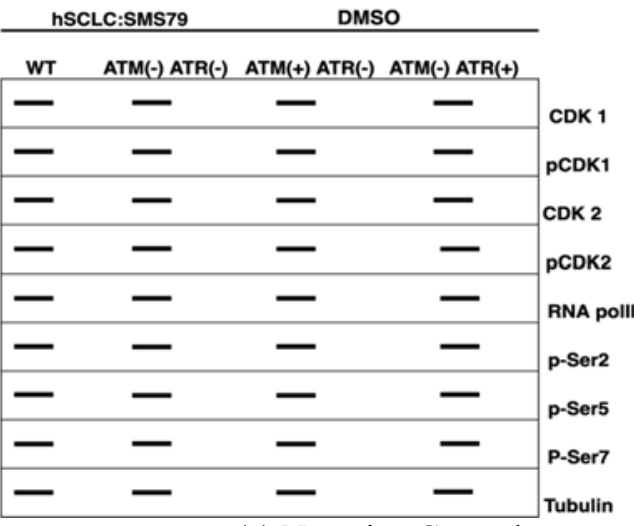

(a) Negative Control effective biomarkers for early detection of DSBs [34]. The phosphorylation of H2AX can achieve by various pathways, such as ATM and DNA-dependent protein kinase (DNA-PK) [35]. Thus, $\gamma \mathrm{H} 2 \mathrm{AX}$ foci formation increased upon YKL-5-124 exposure in wild-type cell lines samples, but rose to a lesser extent in various ATM/ATR- deficient cell lines, revealing action of DNA damage response. Although $\mathrm{CDK} 7$ is deemed to phosphorylate the Ser-5/7 in RNA pol II CTD, YKL-5124 did not down-regulate Ser5/7/2 phosphorylation (Fig. 4).

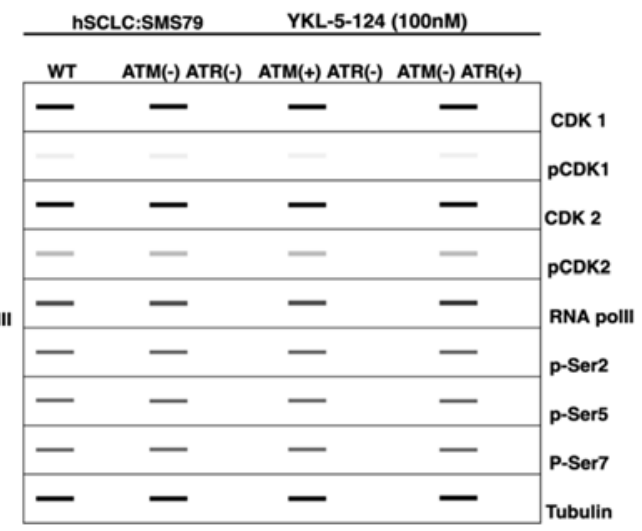

(b) Positive Control

Fig.4 Sample Western blotting of CDK 1, pCDK1 (Thr161), CDK2, pCDK2 (Thr160), RNA pol II, pSer2/5/7, and Tubulin in human SCLC DMS79 cells after treatment DMSO (negative control) and YKL-5-124 (positive control) at $100 \mathrm{nM}$ for $24 \mathrm{~h}$.

\subsection{Effects of ATM and ATR protein on SCLC cell lines survival measure by MTS-based CCK-8 assay (Cell viability). Western Blot validates ATM and ATR protein expression.}

Table 3 Cell viability after knocking-down ATM or ATR mRNA expression with shRNA. "+” represents cell alive, and “-” represents cell death.

\begin{tabular}{|c|c|c|c|c|}
\hline Possible Result & $\begin{array}{c}\text { WT: ATM (present) } \\
\text { ATR(present) }\end{array}$ & $\begin{array}{c}\text { ATM } \\
\text { (knocked- } \\
\text { down) ATR } \\
\text { (knocked- } \\
\text { down) }\end{array}$ & $\begin{array}{c}\text { ATM (present) } \\
\text { ATR (knocked- } \\
\text { down) }\end{array}$ & $\begin{array}{c}\text { ATM } \\
\text { (knocked- } \\
\text { down) ATR } \\
\text { (present) }\end{array}$ \\
\hline 1 & + & - & - & - \\
\hline
\end{tabular}




\begin{tabular}{|c|c|c|c|c|}
\hline 2 & + & - & - & + \\
\hline 3 & + & - & + & - \\
\hline 4 & + & + & + & + \\
\hline
\end{tabular}

Possible result 1: Both ATM and ATR proteins are essential for purified SCLC cell lines samples' survival.

Possible result 2: Only ATR protein is essential for purified SCLC cell lines samples' survival.

Possible result 3: Only ATM protein is essential for purified SCLC cell lines samples' survival.
Possible result 4: ATM and ATR proteins are not essential for purified SCLC cell lines samples' survival.

If ATM and ATR proteins are not essential for SCLC cell lines samples' survival, Western Blot is required to validate ATR and ATM proteins expression (Fig 5).

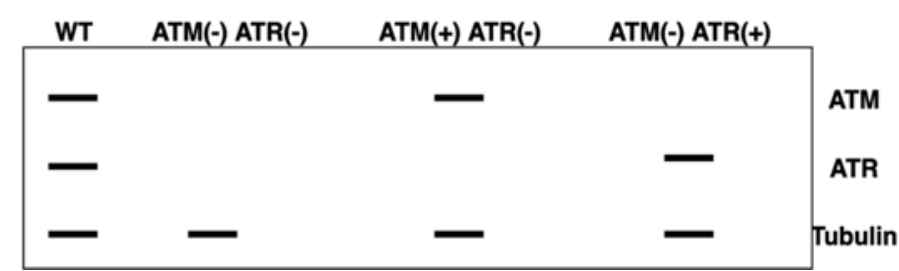

Fig.5 Western Blot analysis: Validation of ATR and ATM are not essential for purified SCLC cell lines samples' survival.

\subsection{If ATM and ATR proteins are not essential for SCLC cell lines samples' survival. Possible results on the relationship between ATR and ATM and inflammatory factor production due to CDK7 inhibition to purified SCLC cell lines by YKL-5-124.}

After the SCLC cells are purified and successfully knocked down by shRNA, they are separated into positive control and negative control. The purified SCLC cells are treated with YKL-5-124 in the positive control group and DMSO in the negative control group. Besides, since IL- 8 and TGF - $\beta$ are both major factors of SASP and cytokines produced by most SCLC cells (Fig.1), the mRNA and protein expression levels of IL8 and TGF- $\beta$ will be measured in both a positive control and negative control in this control experiment. Meanwhile, since more than one DNA repair pathway may secrete cytokines and not be limited to IL8 and TGF- $\beta$, measuring other cytokine expressions to verify diverse pathways in response to DNA damage is necessary. As Zhang et al. noted, the increased micronucleus formation is associated with immune-response activation [10]. This experiment will explore whether the increase of micronucleus directly leads to cytokine secretion in purified SCLC cells. Or, the generation of micronucleus is just a byproduct of DNA damage. Later IL8, TGF- $\beta$, other cytokines expression, micronucleus, and $\gamma \mathrm{H} 2 \mathrm{AX}$ formation in positive control and negative control are compared. The table is shown as follow:

Table 4 IL8, TGF- $\beta$, other cytokines expression, micronucleus, and $\gamma \mathrm{H} 2 \mathrm{AX}$ formation comparison after treating the purified SCLC cell lines with 100nM YKL-5-124 and DMSO. "+" represents increased signal detection compared with the DMSO controls; "“-" represents decreased signal detection compared with DMSO controls, and " 0 " represents no detection compared with DMSO controls. (“*”" indicates the same phenotype with different interpretation)

\begin{tabular}{|c|c|c|c|c|c|c|c|c|c|c|}
\hline \multicolumn{11}{|c|}{ Possible Results } \\
\hline & & 5 & 6 & 7 & $8^{*}$ & 9 & $10^{*}$ & 11 & 12 & 13 \\
\hline \multirow[t]{5}{*}{ WT } & IL-8 & + & + & + & + & + & + & 0 & 0 & + \\
\hline & TGF- $\beta$ & + & + & + & + & + & + & 0 & 0 & + \\
\hline & $\begin{array}{l}\text { Other } \\
\text { cytokines }\end{array}$ & 0 & 0 & 0 & 0 & + & 0 & + & 0 & 0 \\
\hline & Micronucleus & + & + & + & + & + & + & + & + & 0 \\
\hline & $\gamma \mathrm{H} 2 \mathrm{AX}$ & +++ & +++ & +++ & +++ & +++ & +++ & +++ & +++ & +++ \\
\hline \multirow{2}{*}{$\begin{array}{l}\text { ATM } \\
(-) \\
\text { ATR (- }\end{array}$} & IL-8 & 0 & 0 & 0 & + & + & + & 0 & 0 & 0 \\
\hline & TGF- $\beta$ & 0 & 0 & 0 & + & + & + & 0 & 0 & 0 \\
\hline
\end{tabular}




\begin{tabular}{|c|c|c|c|c|c|c|c|c|c|c|}
\hline \multirow[t]{3}{*}{ ) } & $\begin{array}{l}\text { Other } \\
\text { cytokines }\end{array}$ & 0 & 0 & 0 & 0 & + & 0 & + & 0 & 0 \\
\hline & Micronucleus & + & + & + & + & + & + & + & + & 0 \\
\hline & $\gamma \mathrm{H} 2 \mathrm{AX}$ & + & + & + & + & + & + & + & + & + \\
\hline \multirow{5}{*}{$\begin{array}{l}\text { ATM } \\
(+) \\
\text { ATR (- } \\
)\end{array}$} & IL-8 & 0 & + & 0 & + & + & + & 0 & 0 & 0 \\
\hline & TGF- $\beta$ & 0 & + & 0 & + & + & + & 0 & 0 & 0 \\
\hline & $\begin{array}{l}\text { Other } \\
\text { cytokines }\end{array}$ & 0 & 0 & 0 & 0 & + & 0 & + & 0 & 0 \\
\hline & Micronucleus & + & + & + & + & + & + & + & + & 0 \\
\hline & $\gamma \mathrm{H} 2 \mathrm{AX}$ & ++ & ++ & ++ & ++ & ++ & ++ & ++ & ++ & ++ \\
\hline \multirow{5}{*}{$\begin{array}{l}\text { ATM } \\
(-) \\
\text { ATR } \\
(+)\end{array}$} & IL-8 & 0 & 0 & + & + & + & + & 0 & 0 & 0 \\
\hline & TGF- $\beta$ & 0 & 0 & + & + & + & + & 0 & 0 & 0 \\
\hline & $\begin{array}{l}\text { Other } \\
\text { cytokines }\end{array}$ & 0 & 0 & 0 & 0 & + & 0 & + & 0 & 0 \\
\hline & Micronucleus & + & + & + & + & + & + & + & + & 0 \\
\hline & $\gamma \mathrm{H} 2 \mathrm{AX}$ & ++ & ++ & ++ & ++ & ++ & ++ & ++ & ++ & ++ \\
\hline \multicolumn{2}{|c|}{ Support Hypothesis } & +++ & ++ & ++ & - & -- & - & - & - & ++++ \\
\hline
\end{tabular}

Possible result 5: Both ATM and ATR proteins are necessary to trigger inflammatory cytokine secretion by inducing the SASP factor in responding to DNA damage caused by CDK7 inhibition by YKL-5-124 in purified SCLC cell lines. Meanwhile, micronucleus formation is a DNA damage indicator.

Possible result 6: Only ATM protein is necessary to trigger inflammatory cytokine secretion by inducing the SASP factor in responding to DNA damage caused by CDK7 inhibition by YKL-5-124 in purified SCLC cell lines. Meanwhile, micronucleus formation is a DNA damage indicator.

Possible result 7: Only ATR protein is necessary to trigger inflammatory cytokine secretion by inducing the SASP factor in responding to DNA damage caused by CDK7 inhibition by YKL-5-124 in purified SCLC cell lines. Meanwhile, micronucleus formation is a DNA damage indicator.

Possible result 8: There are other pathways besides ATM and ATR DNA damage repair pathways that trigger inflammatory cytokine secretion by inducing the SASP factor in responding to DNA damage caused by CDK7 inhibition by YKL-5-124 in purified SCLC cell lines. Meanwhile, micronucleus formation is a DNA damage indicator.

Possible result 9: ATM and ATR cooperate with other DNA damage repair pathways that trigger various inflammatory cytokines secretion in responding to DNA damage caused by CDK7 inhibition by YKL-5-124 in purified SCLC cell lines. Meanwhile, micronucleus formation is a DNA damage indicator.

Possible result 10: Micronucleus formation triggers inflammatory cytokine secretion by inducing the SASP factor in responding to DNA damage caused by CDK7 inhibition by YKL-5-124 in purified SCLC cell lines.

Possible result 11: Other pathways besides (or cooperate with) ATM and ATR DNA damage repair pathways that trigger inflammatory cytokine secretion in purified SCLC cell lines samples by inducing inflammatory cytokines other than the IL-8 and TGF- $\beta$ in responding to DNA damage. Meanwhile, micronucleus formation is a DNA damage indicator.

Possible result 12: CDK7 inhibition by YKL-5-124 caused DNA damage. Neither ATM/ATR DNA damage repair pathways nor micronucleus formation induced inflammatory cytokines secretion purified SCLC cell lines.

Possible result 13: Both ATM and ATR proteins are necessary to trigger inflammatory cytokine secretion by inducing the SASP factor in responding to DNA damage caused by CDK7 inhibition by YKL-5-124 in purified SCLC cell lines. However, there is no micronucleus formation.

Possible result 14: YKL-5-124 triggers inflammatory cytokine secretion in purified SCLC cell lines sample by inducing the SASP factor in responding to DNA damage. However, human and mouse cell lines have different levels of secretion.

\section{Discussion}

In this proposal, experiments are required to confirm YKL-5-124 inhibits CDK7 in purified SCLC cells and impairs DNA replication to ensure the following experiment is based upon Zhang et al.'s experiment. 


\subsection{Based on phenotypes, 14 possible results can be subcategorized into eight groups. The explanations for each group are provided below:}

(1) Possible results 1 to 3: These results are required to set up the subsequent experiments to test the hypothesis. Group 1 result validates knocking down ATM and ATR proteins with shRNA not affecting purified SCLC cells' survival and allowing further investigations.

(2) Possible results 5 and 13: Result 5 strongly supports the hypothesis. In terms of result 5, IL-8 and TGF- $\beta$ are not produced in the negative control nor two perturbations. The wild-type secretes both IL-8 and TGF$\beta$, which reveals both ATM and ATR signaling pathways are necessary to repair DNA damage caused by CDK7 inhibition by YKL-5-124 in purified SCLC cells and trigger inflammatory cytokines secretion via inducing the SASP. However, micronucleus is detected in all the controls. There is not enough evidence to suggest that micronucleus is not a cause of cytokine formation. On the other hand, the result 13 completely supports the hypothesis. It suggests both ATM and ATR pathways are necessary to secret SASP factors (IL-8 and TGF- $\beta$ ) while no micronucleus formation is detected.

(3) Possible results 6 and 7: These results partially support the hypothesis. Result 6 and 7 suggest that the combination of ATM and ATR pathways is not needed. Instead, ATM or ATR signaling pathway alone may be activated by DNA damage caused by CDK 7 inhibition in purified SCLC cells and trigger inflammatory cytokine(s) secretion by inducing the SASP factor. Likewise, there is not enough evidence to support micronucleus is not a cause of cytokine formation in these two possible results.

(4) Possible results $8^{*}, 9,10^{*}, 11$, and 12: These four results do not support the hypothesis. Results 8,9 , and 11 suggest other signaling pathways exist besides ATM and ATR. Different possible pathways also have the ability to secrete cytokines, which are not limited to SASP factors in response to DNA damage (result 9 and 11). Intriguingly, result 11 might also suggest that both ATM and ATR signaling pathways are necessary to trigger SASP and other signaling pathways exist besides ATM and ATR secreting different types of cytokines. In both results 8 and 9, micronucleus formation is observed in all the controls. There is not enough evidence to suggest micronucleus is not a cause of cytokine formation. Nevertheless, result 12 supports that micronucleus is a DNA damage indicator, although it suggests SCLC cells do not secrete cytokine in response to DNA damage. Interestingly, result 10 has the same phenotype as result 8 , yet it comes with different interpretations. Result 10 can also be interpreted as micronucleus is a cause of SASP factors secretion in response to DNA damage.

(5) Possible result 14: This result does not support the hypothesis, which might be due to the difference in biological systems between humans and mice.

These possible experimental results are closely related to the various possibilities of CDK7 inhibition by YKL-5-124 on SCLC cells' molecular mechanisms. Although results 5 to 12 do not support that micronucleus is not a cause of cytokine formation, it is reasonable to believe that results from 5 to 12 could be observed. Result 13 completely supports the hypothesis, but micronucleus formation is almost always detected along with DNA damage and genome instability.

\subsection{Micronucleus Are the Indicator Only}

Zhang et al. stated that the inhibition of CDK7 by YKL5-124 that triggers cytokine production does not follow the most popular cytosolic DNA-sensing pathway cGAS- STING signaling pathway. The cGAS- STING pathway involves the innate immune response mediated by cytoplasmic DNA [10]. cGAS acts as a DNA receptor that recognizes double-stranded DNA produced by bacteria, viruses, and tumor cells. cGAMP binds and activates STING, changes its conformation, recruits TBK1, and phosphorylated IRF3. Phosphorylated IRF3 enters the nucleus, inducing typelinterferons, and associating with other immune regulation factors of cells $[36,37]$. It is worth noting that cGAS recognizes the phosphodiester backbone of dsDNA through electrostatic interaction. It does not recognize DNA in a sequencespecific manner [38, 39]. Moreover, studies revealed the length of dsDNA affects the activation of cGAS, and when the length of dsDNA is less than $12 \mathrm{bp}$, it cannot activate cGAS effectively [40, 41]. Nevertheless, the SCLC cells were confirmed to secrete cytokine, but not typeI interferons [13]. Therefore, the inhibition of CDK7 by YKL-5-124 in SCLC cells can induce micronucleus production. Still, it cannot support that micronucleus is the direct cause of cytokine induction in SCLC cells, and the results 5 to 12 are likely to be observed. It is because 1) there is no evidence that SCLC cells can produce the type I interferons; 2) dsDNA might not leave micronucleus to the cytosol due to the protection of nuclear lamina, or 3) dsDNA is less than $12 \mathrm{bp}$ and degraded by DnaseI; thus, cGAS-STING pathway could not be activated.

\subsection{Cytokines and Pathway}

Zhang et al. and Olson et al. confirmed that YKL-5-124 inhibits CDK7 effectively and arrests the cell cycle that induces genomic stability in SCLC cells. Zhang et al. suggested that the inhibition of CDK7 by YKL-5-124 could increase IFN- $\gamma$, TNF- $\alpha$, and inflammatory responses in SCLC cells (result 9 and 11) [10]. However, there is no evidence suggesting SCLC cells secrete IFN- $\gamma$ (a type of type I interferons) nor TNF- $\alpha$ [14]. The type I interferons are also mainly induced by the cGAS-STING pathway. Interestingly, IL-8 and TGF - $\beta$ are both produced in SCLC cell lines [13]. Meanwhile, IL-8 and TGF - $\beta$ also belong to the SASP factor secreted by the senescent cells. Cell damage does not directly lead to signs of aging; however, when the damage accumulates and reaches to a certain point, the cell proliferation stops, leading to a physiological aging phenotype [41]. Thus, the CDK7 inhibition by YKL-5-124 might cause the purified SCLC cells to senesce and secrete SASP (result 5-9 and 13). Zhang et al. and Olson et al. demonstrated that YKL-5-124 inhibited CDK2 phosphorylation in Jurkat and SCLC cells at a different level, reduced the 
number of cells in the S phase, but SCLC cells are cumulated in G1 phase [10-11]. The increasing $\gamma \mathrm{H} 2 \mathrm{AX}$ (All the results) suggested DNA damage upon YKL-5124 exposure in SCLC cells (result 5-13). Besides, treating SCLC cells with YKL-5-124 decreased M2M numbers [10], which reveals most SCLC cells arrested in the late G1 phase and ARS (Autonomously Replication Site) are occupied that MCM2-7 cannot bind to ARS, and they were phosphonate and sent out of the nucleus.

On the other hand, cell senescence also occurs in damaged cells. Although SASP is a common feature of senescent cells, the SASP components are different in various kinds of aging cells or other stimulation-induced senescence cells (Result 11 and 14). Some aging inducing factors (including DNA damage and carcinogenic mutation) can act on cells alone or in combination, including ATM and ATR kinase signal transduction, leading to cell senescence through p16INK4a / RB, p53 / p21 pathway or other pathways (Result 6-9,11 and 12) [42]. Activation of DDRs in senescent cells promotes the acquisition of SASP. Meanwhile, miRNAs are also related to senescent cell regulation and DDR inflammation [43].

\section{Conclusion}

Zhang et al. used YKL-5-124; a CDK7 inhibitor demonstrates that inhibition of CDK7 disrupted the cell cycle. Inhibition leads to DNA replication pressure and genomic instability in SCLC cells, which further triggers the signal transduction of immune response [10]. It is worth noting that SCLC cells are proposed to be purified in this paper, not mentioned in Zhang's experiment. Therefore, the cytokines IFN- $\gamma$ they detected may be produced by immune cells in the contaminated SCLC cells.

Further studies are needed to test the efficacy of YKL-5-124 and anti-PD-L1 in other types of cancer. More investigations are required to unmask the molecular interaction between the inhibited CDK7 and CDK2 phosphorylation in SCLC cells, as well as exploring the specific mechanism that CDK7 regulates basal transcription.

More importantly, since the pathway in which CDK7 inhibition by YKL-5-124 induces cytokines production in SCLC remains unknown. The result of this study will reveal the effects of CDK7 inhibition by YKL-5-124 on the molecular mechanisms of SCLC and potentially promote the development of new anticancer treatment strategies based on immunity manipulation, which is a dawn of future cancer treatments.

\section{Acknowledgments}

This paper was completed under the guidance of Professor Arthur $r$ Salomon, Brown University. In this paper, I encountered many problems, which were solved under Professor Salomon's patient guidance. Here, I would like to express my high respect and heartfelt thanks to Professor Salomon.

\section{Reference}

1. Pietanza, M. C., Byers, L. A., Minna, J. D., \& Rudin, C. M. (2015). Small cell lung cancer: will recent progress lead to improved outcomes?. Clinical cancer research : an official journal of the American Association for Cancer Research, 21(10), 22442255.

2. Hanahan, D., \& Weinberg, R. A. (2000). The hallmarks of cancer. cell, 100(1), 57-70.

3. Lrochelle, S., Chen, J., Knights, R., Pandur, J., Morcillo, P., Erdjument-Bromage, H., ... \& Fisher, R. P. (2001). T-loop phosphorylation stabilizes the CDK7-cyclin H-MAT1 complex in vivo and regulates its $\mathrm{CTD}$ kinase activity. The EMBO journal, 20(14), 3749-3759.

4. Lolli, G., \& Johnson, L. N. (2005). CAK-cyclindependent activating kinase: a key kinase in cell cycle control and a target for drugs?. Cell cycle, 4(4), 565-570.

5. Ebmeier, C., Erickson, B., Allen, B., Allen, M., Kim, H., Fong, N., ... \& Old, W. (2018). Human TFIIH Kinase CDK7 Regulates Transcription-Associated Epigenetic Modifications. Available at SSRN 3155590.

6. Parua, P. K., \& Fisher, R. P. (2020). Dissecting the Pol II transcription cycle and derailing cancer with CDK inhibitors. Nature chemical biology, 16(7), 716-724.

7. Yankulov, K. Y., \& Bentley, D. L. (1997). Regulation of CDK7 substrate specificity by MAT1 and TFIIH. The EMBO journal, 16(7), 1638-1646.

8. Ganuza, M., Sáiz-Ladera, C., Cañamero, M., Gómez, G., Schneider, R., Blasco, M. A., . . . Barbacid, M. (2012). Genetic inactivation of $\mathrm{Cdk} 7$ leads to cell cycle arrest and induces premature aging due to adult stem cell exhaustion. The EMBO Journal, 31(11), 2498-2510.

9. Kanin, E. I., Kipp, R. T., Kung, C., Slattery, M., Viale, A., Hahn, S., ... \& Ansari, A. Z. (2007). Chemical inhibition of the TFIIH-associated kinase Cdk7/Kin28 does not impair global mRNA synthesis. Proceedings of the National Academy of Sciences, 104(14), 5812-5817.

10. Zhang, H., Christensen, C. L., Dries, R., Oser, M. G., Deng, J., Diskin, B., ... \& Papadopoulos, E. (2020). CDK7 inhibition potentiates genome instability triggering anti-tumor immunity in small cell lung cancer. Cancer Cell, 37(1), 37-54.

11. Olson, C. M., Liang, Y., Leggett, A., Park, W. D., Li, L., Mills, C. E., ... \& Geyer, M. (2019). Development of a selective CDK7 covalent inhibitor reveals predominant cell-cycle phenotype. Cell chemical biology, 26(6), 792-803.

12. Christensen, C. L., Kwiatkowski, N., Abraham, B. J., Carretero, J., Al-Shahrour, F., Zhang, T., ... \& Zhang, J. (2014). Targeting transcriptional addictions in small cell lung cancer with a covalent CDK7 inhibitor. Cancer cell, 26(6), 909-922.

13. Fukuyama, T., Ichiki, Y., Yamada, S., Shigematsu, Y., Baba, T., Nagata, Y., ... \& Sugio, K. (2007). Cytokine production of lung cancer cell lines: 
Correlation between their production and the inflammatory/immunological responses both in vivo and in vitro. Cancer Science, 98(7), 1048-1054.

14. Ovadya, Y., \& Krizhanovsky, V. (2014). Senescent cells: SASPected drivers of age-related pathologies. Biogerontology, 15(6), 627-642.

15. Petroni, G., Formenti, S. C., Chen-Kiang, S., \& Galluzzi, L. (2020). Immunomodulation by anticancer cell cycle inhibitors. Nature Reviews Immunology, 1-11.

16. Malaquin, N., Carrier-Leclerc, A., Dessureault, M., \& Rodier, F. (2015). DDR-mediated crosstalk between DNA-damaged cells and their microenvironment. Frontiers in genetics, 6, 94.

17. Coppé, J. P., Desprez, P. Y., Krtolica, A., \& Campisi, J. (2010). The senescence-associated secretory phenotype: the dark side of tumor suppression. Annual Review of Pathology: Mechanisms of Disease, 5, 99-118.

18. Morsy, M. A., Norman, P. J., Mitry, R., Rela, M., Heaton, N. D., \& Vaughan, R. W. (2005). Isolation, purification and flow cytometric analysis of human intrahepatic lymphocytes using an improved technique. Laboratory investigation, 85(2), 285-296.

19. Rawstron, A. C. (2006). Immunophenotyping of plasma cells. Current protocols in cytometry, 36(1), 6-23.

20. Oleinika, K., Mauri, C., \& Salama, A. D. (2019). Effector and regulatory B cells in immune-mediated kidney disease. Nature Reviews Nephrology, 15(1), 11-26.

21. Cameron, A. L., Kirby, B., \& Griffiths, C. E. M. (2003). Circulating natural killer cells in psoriasis. British Journal of Dermatology, 149(1), 160-164.

22. Solano-Gálvez, S. G., Tovar-Torres, S. M., TronGómez, M. S., Weiser-Smeke, A. E., ÁlvarezHernández, D. A., Franyuti-Kelly, G. A., ... \& Vázquez-López, R. (2018). Human dendritic cells: ontogeny and their subsets in health and disease. Medical Sciences, 6(4), 88.

23. Chen, K., Liu, J., Heck, S., Chasis, J. A., An, X., \& Mohandas, N. (2009). Resolving the distinct stages in erythroid differentiation based on dynamic changes in membrane protein expression during erythropoiesis. Proceedings of the National Academy of Sciences, 106(41), 17413-17418.

24. Kim, M. Y., Yu, K. R., Kenderian, S. S., Ruella, M., Chen, S., Shin, T. H., ... \& Kozlowski, M. S. (2018). Genetic inactivation of CD33 in hematopoietic stem cells to enable CAR T cell immunotherapy for acute myeloid leukemia. Cell, 173(6), 1439-1453.

25. Sano, K., Yamauchi, K., Hoshi, H., Honma, M., Tamura, G., \& Shirato, K. (1997). CD44 expression on blood eosinophils is a novel marker of bronchial asthma. International archives of allergy and immunology, 114(Suppl. 1), 67-71.

26. Gustafson, M. P., Lin, Y., Maas, M. L., Van Keulen, V. P., Johnston, P. B., Peikert, T., ... \& Dietz, A. B. (2015). A method for identification and analysis of non-overlapping myeloid immunophenotypes in humans. PloS one, 10(3), e0121546.
27. Collin, M., \& Bigley, V. (2018). Human dendritic cell subsets: an update. Immunology, 154(1), 3-20.

28. Agis, H., Beil, W. J., Bankl, H. C., Füreder, W., Sperr, W. R., Ghannadan, M., ... \& Valent, P. (1996). Mast Cell-Lineage Versus Basophil Lineage Involvement in Myeloproliferative and Myelodysplastic Syndromes: Diagnostic Role of Cell-Immunopheno typing. Leukemia \& lymphoma, 22(3-4), 187-204.

29. Sun, Q., Woodcock, J. M., Rapoport, A., Stomski, F. C., Korpelainen, E. I., Bagley, C. J., ... \& Lopez, A. F. (1996). Monoclonal antibody $7 \mathrm{G} 3$ recognizes the $\mathrm{N}$-terminal domain of the human interleukin-3 (IL-3) receptor alpha-chain and functions as a specific IL-3 receptor antagonist.

30. Dahl, C., Hoffmann, H. J., Saito, H., \& Schiøtz, P. O. (2004). Human mast cells express receptors for IL-3, IL-5 and GM-CSF; a partial map of receptors on human mast cells cultured in vitro. Allergy, 59(10), 1087-1096.

31. Farber, D. L., Yudanin, N. A., \& Restifo, N. P. (2014). Human memory $\mathrm{T}$ cells: generation, compartmentalization and homeostasis. Nature Reviews Immunology, 14(1), 24-35.

32. Cui, Y., Palii, S. S., Innes, C. L., \& Paules, R. S. (2014). Depletion of ATR selectively sensitizes ATM-deficient human mammary epithelial cells to ionizing radiation and DNA-damaging agents. Cell Cycle, 13(22), 3541-3550.

33. Vendetti, F. P., Lau, A., Schamus, S., Conrads, T. P., O'Connor, M. J., \& Bakkenist, C. J. (2015). The orally active and bioavailable ATR kinase inhibitor AZD6738 potentiates the anti-tumor effects of cisplatin to resolve ATM-deficient non-small cell lung cancer in vivo. Oncotarget, 6(42), 44289.

34. Tu, W. Z., Li, B., Huang, B., Wang, Y., Liu, X. D., Guan, H., ... \& Zhou, P. K. (2013). $\gamma \mathrm{H} 2 \mathrm{AX}$ foci formation in the absence of DNA damage: mitotic $\mathrm{H} 2 \mathrm{AX}$ phosphorylation is mediated by the DNAPKcs/CHK2 pathway. FEBS letters, 587(21), 34373443.

35. Stiff, T., O’Driscoll, M., Rief, N., Iwabuchi, K., Löbrich, M., \& Jeggo, P. A. (2004). ATM and DNA-PK function redundantly to phosphorylate $\mathrm{H} 2 \mathrm{AX}$ after exposure to ionizing radiation. Cancer research, 64(7), 2390-2396.

36. Zhang, Z., Yuan, B., Bao, M., Lu, N., Kim, T., \& Liu, Y. J. (2011). The helicase DDX41 senses intracellular DNA mediated by the adaptor STING in dendritic cells. Nature immunology, 12(10), 959965.

37. Unterholzner, L., Keating, S. E., Baran, M., Horan, K. A., Jensen, S. B., Sharma, S., ... \& Fitzgerald, K. A. (2010). IFI16 is an innate immune sensor for intracellular DNA. Nature immunology, 11(11), 9971004.

38. Sun, L., Wu, J., Du, F., Chen, X., \& Chen, Z. J. (2013). Cyclic GMP-AMP synthase is a cytosolic DNA sensor that activates the type I interferon pathway. Science, 339(6121), 786-791.

39. Li, X., Shu, C., Yi, G., Chaton, C. T., Shelton, C. L., Diao, J., ... \& Li, P. (2013). Cyclic GMP-AMP 
synthase is activated by double-stranded DNAinduced oligomerization. Immunity, 39(6), 10191031.

40. Shu, C., Li, X., \& Li, P. (2014). The mechanism of double-stranded DNA sensing through the cGASSTING pathway. Cytokine \& growth factor reviews, 25(6), 641-648.

41. Byun, H. O., Lee, Y. K., Kim, J. M., \& Yoon, G. (2016). Erratum to: From cell senescence to agerelated diseases: differential mechanisms of action of senescence-associated secretory phenotypes. $B M B$ Rep, 49, 641-650.

42. Tchkonia, T., Zhu, Y., Van Deursen, J., Campisi, J., \& Kirkland, J. L. (2013). Cellular senescence and the senescent secretory phenotype: therapeutic opportunities. The Journal of clinical investigation, 123(3), 966-972.

43. Olivieri, F., Albertini, M. C., Orciani, M., Ceka, A., Cricca, M., Procopio, A. D., \& Bonafè, M. (2015). DNA damage response (DDR) and senescence: shuttled inflamma-miRNAs on the stage of inflammaging. Oncotarget, 6(34), 35509. 